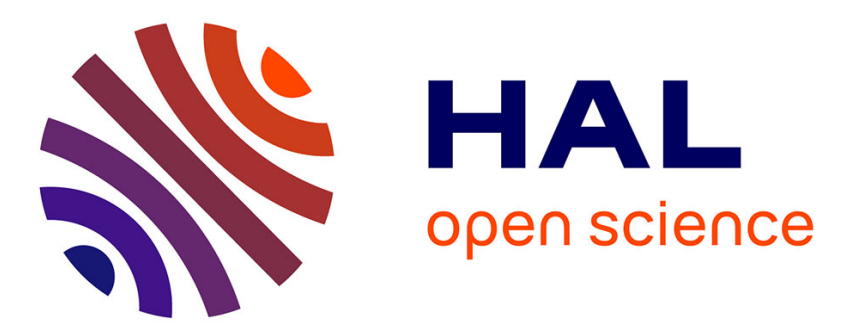

\title{
Simultaneous spatial and temporal focusing: A route towards confined nonlinear materials processing
}

Robert Kammel, Klaus Bergner, Jens Thomas, Roland Ackermann, Stefan Skupin, Stefan Nolte

\section{- To cite this version:}

Robert Kammel, Klaus Bergner, Jens Thomas, Roland Ackermann, Stefan Skupin, et al.. Simultaneous spatial and temporal focusing: A route towards confined nonlinear materials processing. Laser-based Micro- and Nanoprocessing X, Feb 2016, San Francisco, CA, United States. pp.97360T, 10.1117/12.2209540 . hal-01291931

\section{HAL Id: hal-01291931 \\ https://hal.science/hal-01291931}

Submitted on 22 Mar 2016

HAL is a multi-disciplinary open access archive for the deposit and dissemination of scientific research documents, whether they are published or not. The documents may come from teaching and research institutions in France or abroad, or from public or private research centers.
L'archive ouverte pluridisciplinaire HAL, est destinée au dépôt et à la diffusion de documents scientifiques de niveau recherche, publiés ou non, émanant des établissements d'enseignement et de recherche français ou étrangers, des laboratoires publics ou privés. 


\title{
Simultaneous spatial and temporal focusing: A route towards confined nonlinear materials processing
}

\author{
Robert Kammel ${ }^{* a}$, Klaus Bergner ${ }^{\mathrm{a}}$, Jens U. Thomas ${ }^{\mathrm{a}}$, Roland Ackermann ${ }^{\mathrm{a}}$, \\ Stefan Skupin ${ }^{\mathrm{b}}$ and Stefan Nolte ${ }^{\mathrm{ac}}$ \\ ${ }^{a}$ Institute of Applied Physics, Abbe Center of Photonics, Friedrich Schiller University Jena, \\ Albert-Einstein-Straße 15, 07745 Jena, Germany; ${ }^{b}$ Université de Bordeaux - CNRS - CEA, Centre \\ Lasers Intenses et Applications, UMR 5107, 33405 Talence, France; ${ }^{\mathrm{c}}$ Fraunhofer Institute for \\ Applied Optics and Precision Engineering (IOF), Albert-Einstein-Straße 7, 07745 Jena, Germany
}

\begin{abstract}
Ultrashort pulse lasers enable reliable and versatile high precision ablation and surface processing of various materials such as metals, polymers and semiconductors. However, when modifications deep inside the bulk of transparent media are required, nonlinear pulse material interactions can decrease the precision, since weak focusing and the long propagation of the intense pulses within the nonlinear media may induce Kerr self-focusing, filamentation and white light generation. In order to improve the precision of those modifications, simultaneous spatial and temporal focusing (SSTF) allows to reduce detrimental nonlinear interactions, because the ultrashort pulse duration is only obtained at the focus, while outside of the focal region the continuously increasing pulse duration strongly reduces the pulse intensity.

In this paper, we review the fundamental concepts of this technology and provide an overview of its applications for purposes of multiphoton microscopy and laser materials processing. Moreover, numerical simulations on the nonlinear pulse propagation within transparent media illustrate the linear and nonlinear pulse propagation, highlighting the differences between conventional focusing and SSTF. Finally, fs-laser induced modifications in gelatine are presented to compare nonlinear side-effects caused by conventional focusing and SSTF. With conventional focusing the complex interplay of self-focusing and filamentation induces strongly inhomogeneous, elongated disruptions. In contrast, disruptions induced by SSTF are homogeneously located at the focal plane and reduced in length by a factor $>2$, which is in excellent agreement with the numerical simulations of the nonlinear pulse propagation and might favor SSTF for demanding applications such as intraocular fs-laser surgery.
\end{abstract}

Keywords: Simultaneous spatial and temporal focusing, femtosecond laser materials processing, laser-induced optical breakdown, Kerr self-focusing, filamentation, femtosecond laser surgery

\section{INTRODUCTION}

Materials processing using ultrashort femtosecond (fs) laser pulses has gained great attraction within recent years, since new compact and reliable fs-laser sources allow precise and versatile micromachining of numerous materials such as metals or semiconductors. ${ }^{1}$ Due to the short timescales of those pulses, side-effects such as heating of the processed material are hampered, allowing for well-located ablation, drilling or cutting on $\mu \mathrm{m}$-scales. ${ }^{2}$ Moreover, when focused into transparent materials extremely high intensities $>10^{13} \mathrm{~W} / \mathrm{cm}^{2}$ are achieved at the focal region triggering nonlinear absorption processes. ${ }^{3}$ Dependent on the laser parameters and the processed material various types of material modifications can be induced, reaching from well-localized refractive index modifications to extensive disruptions. ${ }^{4}$

In order to gain maximum precision, ultrashort fs-laser pulses are commonly focused with comparatively high numerical aperture (NA) to achieve intensities well above the interaction threshold within a focal spot size of few $\mu \mathrm{m}$. However, for certain applications more sophisticated focusing methods can be required. For instance, the $\mu \mathrm{m}$-sized focal spot provides high spatial and axial resolution in laser-scanning multiphoton fluorescence microscopy, but also demands the timeconsuming scanning of the focal spot across the sample and, thereby, hampers fast image acquisition at video refresh rates.

*robert.kammel@uni-jena.de; phone +49 3641 -9 47 821; fax +49 3641 - 947 802; www.iap.uni-jena.de 
On the other hand weaker focusing with lower NA enables wide-field imaging of the sample area, but suffers from low axial resolution and reduced contrast due to the enlarged Rayleigh length, which also causes the excitation of fluorescent molecules upstream and downstream from the focal plane. Moreover, for various applications such as intraocular fs-laser surgery or back-side processing of glass, ultrashort laser pulses are focused deeply into the material with low NA. ${ }^{5,6}$ As a result, the extreme intensities are not only obtained at the geometrical focus, but may also be achieved already during the propagation towards the focal plane and may evoke nonlinear pulse-material interactions even extending the focal region such as Kerr self-focusing and self-phase modulation of the pulse. ${ }^{7}$ In general these nonlinear interactions are detrimental, since they reduce the precision of the applied modifications and induce side-effects such as strong supercontinuum generation. Moreover, the complex interaction of self-focusing and the defocusing refractive index diminution of the induced plasma can result in the formation of an extended filament, ${ }^{8}$ while intensity clamping inside the filament may impair the development of the material modification. ${ }^{9}$

To overcome those limitations, simultaneous spatial and temporal focusing (SSTF) was proposed as a versatile tool to independently control the size of the focal spot and the corresponding axial resolution. ${ }^{10,11}$ In the concept of SSTF the spectral components of a broadband fs-laser pulse are spatially dispersed outside of the focal volume, which results in a significant increase of the pulse duration. After focusing, the entire pulse spectrum superposes locally at the focal plane and compresses the pulse to its bandwidth-limited pulse duration. Because the ultrashort pulse duration is restricted to the focal plane, the intensity outside of the focal region is strongly reduced, thereby reducing detrimental pulse-material interactions. Moreover, this additional degree of freedom allows shaping the focal intensity distribution. Thus, SSTF has not only raised great attraction for imaging purposes such as multiphoton microscopy, but has also been applied numerously for materials processing such as in-volume structuring of transparent materials. In this publication we provide an insight into the fundamentals of SSTF, present an overview on recent applications in microscopy and material processing, and outline potential future developments of this promising technology.

\section{SIMULTANEOUS SPATIAL AND TEMPORAL FOCUSING IN MICROSCOPY}

High quality multiphoton microscopy requires high lateral and axial resolution together with fast image acquisition. However, with conventional focusing methods it is challenging to address all these demands simultaneously. On the one hand, confocal laser-scanning microscopy provides high resolution and high signal-to-background ratio via excitation of fluorescent molecules within a small $\mu \mathrm{m}$-sized focal volume, but requires a time-consuming scanning of the focal spot across the sample to generate a wide-field image. On the other hand, wide-field imaging with low NA enables imaging the entire sample area at video frame rates without the need of scanning, but suffers from detrimental out-of-focus signal since the increased Rayleigh length causes the excitation of fluorescent molecules outside of the focal plane. In 2005, Zhu et al. ${ }^{10}$ and Oron et al. ${ }^{11}$ proposed SSTF to provide both wide-field imaging with improved depth-resolution and increased signal-to-background ratio. The experimental scheme of this concept is depicted in Figure 1. Like briefly introduced above, an incident fs-laser pulse is spectrally dispersed by means of a reflective grating, which is mounted in the back focal plane of a lens (L1). The spectral separation of the pulse results in a reduction of the local bandwidth within the 'rainbow-like' beam and strongly increases the pulse duration. Thereafter, the stretched pulse is focused by means of

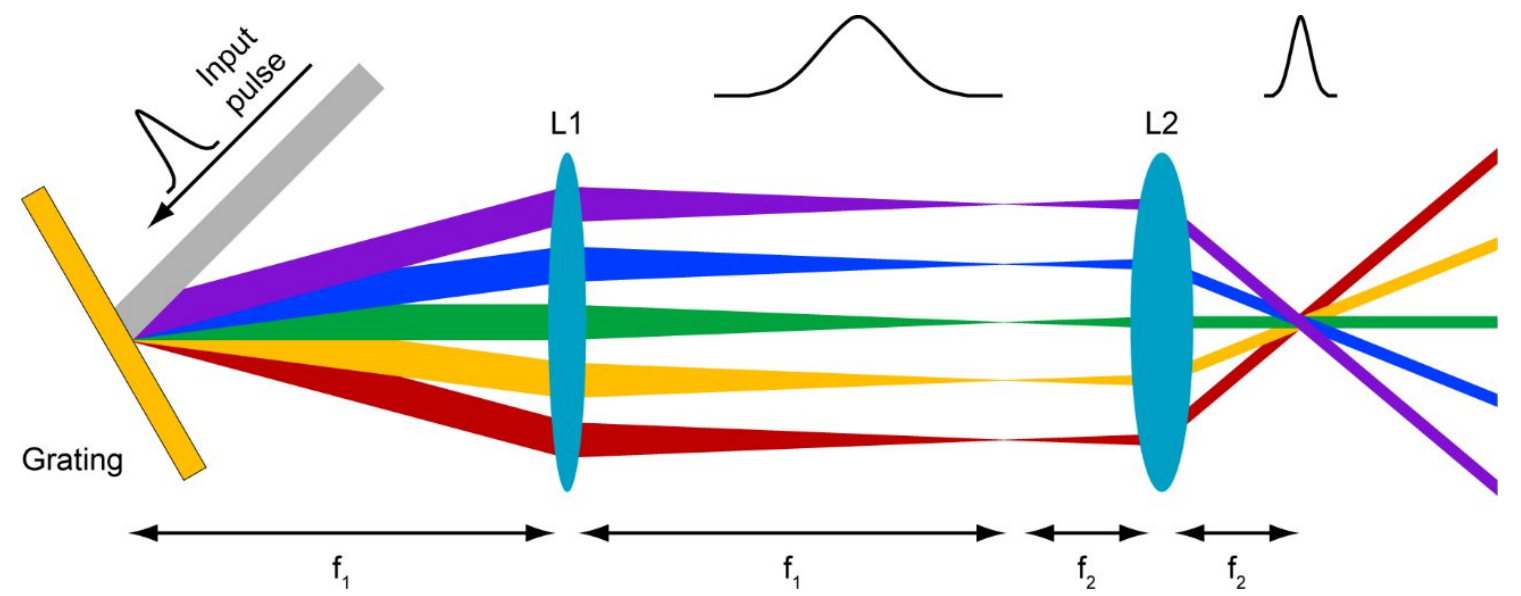

Figure 1. Scheme of simultaneous spatial and temporal focusing applied for wide-field multiphoton microscopy. 
a second lens (L2) or a microscope objective, while the superposition of the entire pulse spectrum causes the compression to the ultrashort pulse duration at the focal plane. Because the ultrashort pulse duration is only achieved at the focus and rapidly increases upstream and downstream from this plane, the nonlinear multiphoton fluorescence signal is strongly restricted to the focal region, thus enabling depth-resolved microscopy with improved signal-to-background contrast. The setup provides wide-field imaging with an enlarged focal spot avoiding the need of scanning the focal spot across the sample, since the focal spot size is determined by the input beam diameter and the magnification of the optical system depicted in Figure 1. ${ }^{12}$

The large potential of this technique has been demonstrated in numerous publications. For example, in the initial paper on SSTF Zhu et al. demonstrated an increase of the pulse duration from $84 \mathrm{fs}$ at the focus to $>1$ ps at a distance of $\pm 200 \mu \mathrm{m}$ away from the focal plane, which was proposed for multiphoton imaging to reduce the out-of-focus background. ${ }^{10}$ Moreover, various innovative procedures made use of the spectrally separated SSTF beam in order to enhance the imaging process. Thus, numerous publications demonstrated the use of spatial light modulators (SLM) providing arbitrarily shaped, depth-resolved excitation patterns to control multiphoton processes with a depth-resolution of few $\mu$ m even at video refresh rates. ${ }^{13-15}$ Similarly, temporally focused computer generated holograms were studied to provide a shift of the temporal focus plane aiming at adapted three-dimensional multiphoton excitation patterns. ${ }^{16}$ Moreover, adding group delay dispersion to the input pulses be means of a deformable mirror device, $\mathrm{z}$-scan with high repetition rate of $200 \mathrm{~Hz}$ for multiphoton microscopy was presented by Straub et al. ${ }^{17}$ In order to further improve the sectioning ability for multiphoton microscopy purposes, Song et al. recently extended the conventional one-dimensional spatial dispersion of SSTF towards a two-dimensional spectral disperser using a virtually imaged phased array..$^{18}$ Due to the enhanced spectral separation ability, both the axial resolution and the image contrast could be significantly enhanced compared to conventional gratingbased SSTF.

\section{SIMULTANEOUS SPATIAL AND TEMPORAL FOCUSING FOR NONLINEAR MATERIALS PROCESSING}

For purposes of multiphoton microscopy, fs-laser pulses are conventionally applied at low energy and high repetition rates up to $\mathrm{MHz}$, while wide-field imaging requires a focal spot size of several tens up to hundreds of $\mu \mathrm{m}$. In contrast, requirements of materials processing in the bulk of transparent media commonly differ from these parameters. For high precision micromachining the pulse energy commonly ranges from few tens up to hundreds of $\mu \mathrm{J}$ to provide intensities $>10^{13} \mathrm{~W} / \mathrm{cm}^{2}$ within a small focal spot size of only few $\mu \mathrm{m}$ in order to trigger nonlinear absorption mechanisms, while repetition rates of several $\mathrm{kHz}$ enable the fast processing of extended regions via scanning the focal spot.

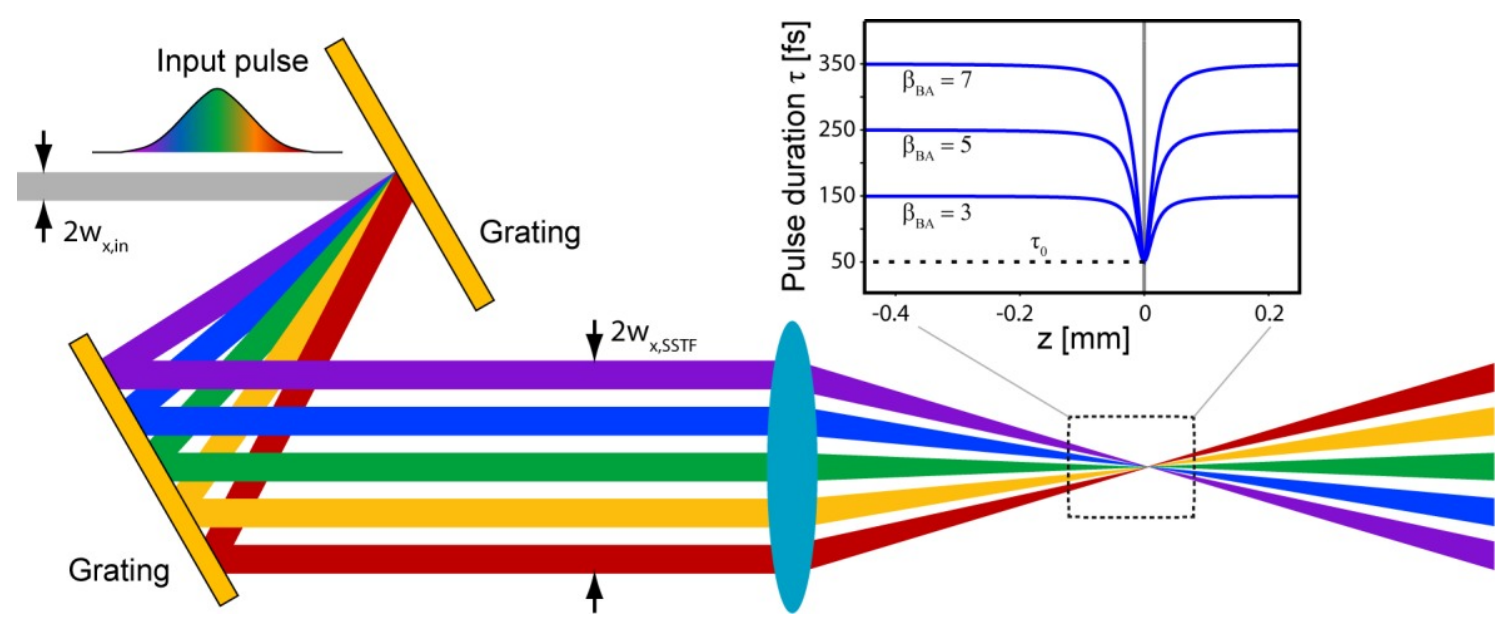

Figure 2. Scheme of simultaneous spatial and temporal focusing based on a single pass parallel grating compressor configuration. The inset illustrates the development of the pulse duration around the focal region depending on the spatial chirp beam aspect ratio $\beta_{\mathrm{BA}}$. 
Hence, SSTF setups used for materials processing are commonly based on single pass parallel grating compressor configurations as shown in Figure 2, which enable restricting the pulse-material interaction to a focal volume of only few $\mu \mathrm{m}$. After passing the grating configuration, the frequency components of the input pulse are linearly displaced by $\alpha \Delta \omega$ along the X-direction depending on the frequency difference $\Delta \omega=\omega-\omega_{0} .{ }^{12}$ Here, $\omega_{0}$ denotes the carrier frequency of the pulse, $\Omega$ is the FWHM of the spectral intensity profile and $\alpha$ describes the spectral separating capability of the grating configuration. After passing the gratings, the pulse with the input beam width $2 w_{x, i n}$ is horizontally stretched to a width of $2 w_{x, S S T F}$. The spectral separation causes an increase of the pulse duration within the collimated SSTF beam to

$$
\tau_{S S T F}=\frac{4 \ln 2}{\Omega} \cdot \sqrt{1+\left(\frac{\alpha \Omega}{w_{x, i n}}\right)^{2}}=\tau_{0} \cdot \beta_{B A} .
$$

Hence, the factor $\beta_{B A}$, by which the transform limited FWHM pulse duration $\tau_{0}$ is increased, raises with increasing spectral separating capability of the grating configuration and decreasing input beam width $2 w_{x, \text { in }}$, since both parameters affect the spectral separation. While the pulse propagates with the FWHM duration $\tau_{S S T F}$ due to the spectral separation, the superposition of the entire bandwidth at the focal plane recompresses the pulse to its bandwidth limited pulse duration $\tau_{0}$ like illustrated in the inset of Figure 2.

In contrast to the single grating configuration presented in Figure 1, the parallel grating setup induces a temporal chirp of the pulse, which is commonly compensated by pre-chirping the input pulse via adjusting the compressor gratings of the laser system or by means of an additional external pulse stretcher setup. To overcome disadvantages such as reduced transmission and the complex alignment of multiple grating configurations, recent efforts aim at incorporating the SSTF grating configuration into chirped pulse amplification (CPA) systems to provide spatially dispersed but temporally unchirped pulses. ${ }^{19,20}$ Due to an adjustable grating configuration, the setups allow tuning the beam aspect ratio $\beta_{\mathrm{BA}}$ to vary the increase of the pulse duration outside of the focal region.

\subsection{Linear and nonlinear propagation of spatially dispersed, focused pulses}

Theoretical treatments of SSTF have shown that the pulse propagation differs from conventional focusing in particular around the focal region. ${ }^{12,21,22}$ To provide a deeper insight into the SSTF pulse propagation, Figure 3 presents x/t-intensity profiles nearby the focal plane comparing pulses focused conventionally and using SSTF. For this numerical simulation, a 'Forward Maxwell equation' (FME) was used to study the linear $\left(\mathrm{E}_{\text {pulse }} \approx 0 \mu \mathrm{J}\right)$ and nonlinear $\left(\mathrm{E}_{\text {pulse }}=4 \mu \mathrm{J}\right)$ pulse propagation within a water cell with a volume of $2 \times 2 \times 4 \mathrm{~mm}^{3}$. A detailed description of this study can be found elsewhere. ${ }^{23}$ To compare SSTF with conventional focusing, a circular beam with a $1 / \mathrm{e}^{2}$-radius of $200 \mu \mathrm{m}$ and an initial pulse duration of $\tau_{0}=50 \mathrm{fs}$, centered at $\lambda_{0}=800 \mathrm{~nm}$, was conventionally focused initially using an ideal lens ( $\left.\mathrm{f}=2 \mathrm{~mm}\right)$ into the center of the observed volume. In contrast, the SSTF pulse was composed of elliptical, pulsed beamlets with $\mathrm{w}_{\mathrm{x}, \mathrm{in}}=40 \mu \mathrm{m}$ and $\mathrm{W}_{\mathrm{y}}=200 \mu \mathrm{m}$. Since the spatial chirp rate $\beta_{\mathrm{BA}}$ was set to 5 , the SSTF pulse duration $\tau_{\mathrm{SSTF}}$ is increased by a factor of 5 to $250 \mathrm{fs}$ before focusing, while the beam is horizontally stretched to a radius of $\mathrm{w}_{\mathrm{x}, \mathrm{SSTF}} \approx 200 \mu \mathrm{m}$. Due to the similar beam size $\left(\mathrm{w}_{\mathrm{x}} \approx \mathrm{w}_{\mathrm{y}} \approx 200 \mu \mathrm{m}\right)$ in front of the ideal focusing lens, $\mathrm{NA} \approx 0.1$ for both regimes.

Regarding conventional focusing for a low pulse energy (linear pulse propagation), the intensity profiles presented in Figure 3a show the well-known symmetrically converging and diverging behavior with a focal spot size of $\sim 5 \mu \mathrm{m}$, while the pulse duration of $\tau_{0}=50 \mathrm{fs}$ is constant during the propagation. In contrast, the pulse propagation of a focused SSTF pulse (Figure 3b) strongly differs from this behavior: Outside of the focal plane, the spectral separation increases the pulse duration to $\tau_{\mathrm{SSTF}}=250 \mathrm{fs}$ ('global pulse duration'). Due to the spectral overlap of the full pulse spectrum nearby the focal plane, the pulse is re-compressed and a strong pulse front tilt (PFT) is induced. Since the PFT causes a fast sweeping of the pulse across the focal plane, the 'local pulse duration' is reduced to $\tau=220 \mathrm{fs}$ at $\mathrm{z}= \pm 200 \mu \mathrm{m}$ and to the bandwidthlimited duration $\tau_{0}=50 \mathrm{fs}$ at $\mathrm{z}=0$, respectively. In contrast, the 'global pulse duration' remains at a constantly increased level of $\tau_{\mathrm{SSTF}} \approx 250 \mathrm{fs}$ during the entire pulse propagation. Although the NA $\approx 0.1$ is similar for both configurations, the horizontal focal spot size is increased by a factor of 5 in case of SSTF due to the elliptical input beamlets described above.

This property of spatially dispersed, focused pulses was experimentally verified using various measurement techniques such as nonlinear fluorescent samples, SEA TADPOLE devices and interferometric characterization. ${ }^{24-26}$ Moreover, numerical analysis of the pulse propagation has shown that the focal PFT is a characteristic feature of SSTF, which cannot 
(a)

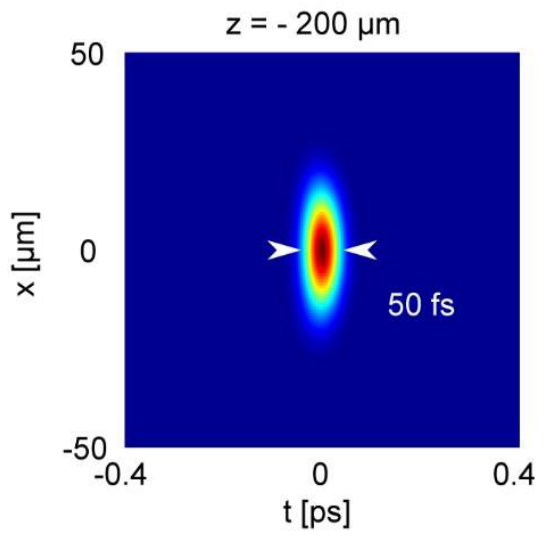

Conventional focusing

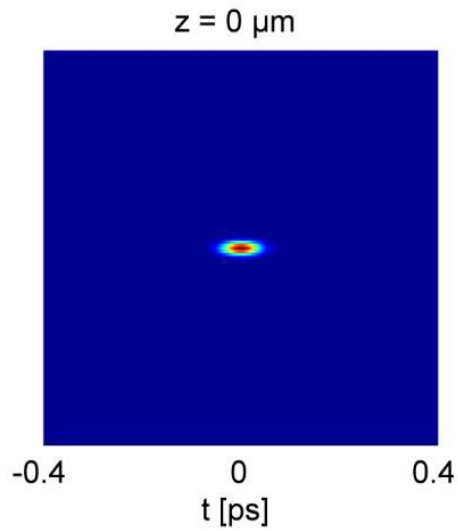

Simultaneous spatial and temporal focusing

(b)
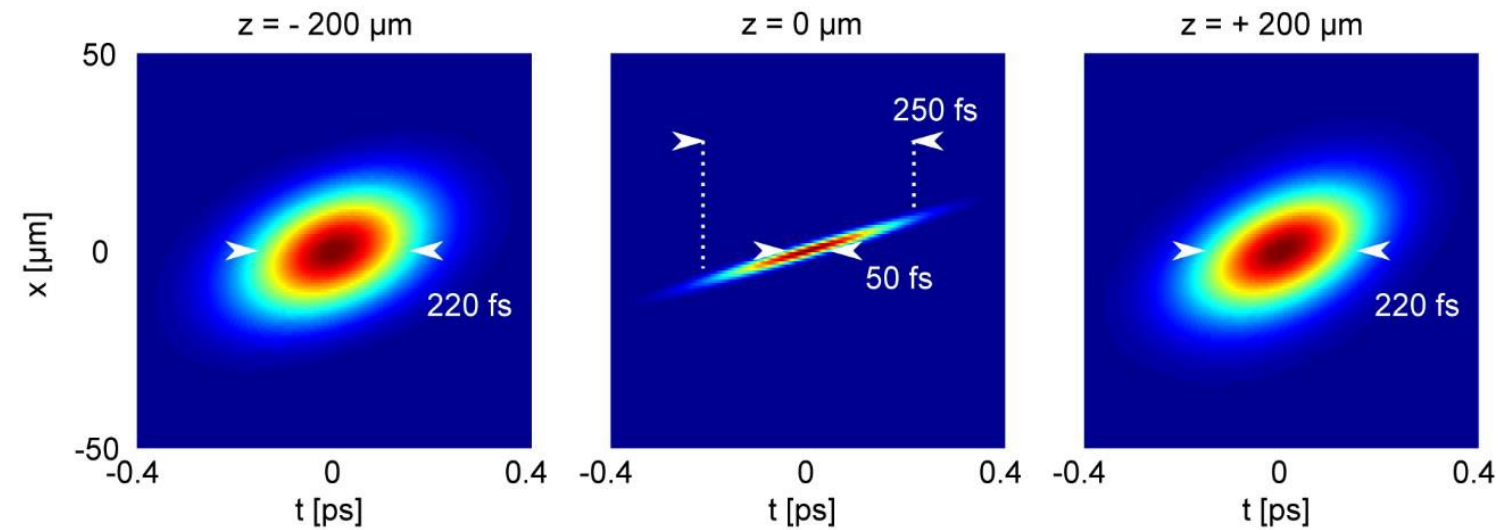

Figure 3. Simulation of the linear pulse propagation $\left(\mathrm{E}_{\text {pulse }} \approx \boldsymbol{0} \boldsymbol{\mu J}\right)$ of pulses focused (a) conventionally and (b) using SSTF, showing $x / t$-intensity profiles nearby the focal plane $(z=-200 \mu \mathrm{m}, 0 \mu \mathrm{m},+200 \mu \mathrm{m})$.

be overcome by techniques such as applying an opposed PFT or pre-chirping the input pulse. ${ }^{22}$ As shown by Kazansky et al. a tilted focal intensity distribution can be used for so-called 'nonreciprocal-' or 'quill writing'. ${ }^{27}$ When focused onto the material surface or into the bulk the orientation of the focal intensity tilt towards the scan direction influences the pulseplasma interaction and the resulting type of material modifications, e.g. for purposes of optical storage, waveguide writing and laser surgery. ${ }^{4}$ Since PFT is an intrinsic feature of spatially dispersed, focused pulses, the formation of wells and Chevron structures through the ablation of glass was demonstrated being dependent on the writing direction of focused SSTF pulses, respectively. ${ }^{28}$

With increasing pulse energy, nonlinear interactions between the field of the incident laser pulse and the transparent medium occur not only at the focal plane, but can induce detrimental side-effects even outside of the focal volume. Namely, self-focusing and filamentation can cause the collapse of the beam far in front of the focal plane and trap the high intensity within a prolonged channel even exceeding the focal plane, while self-phase modulation may cause a self-steepening of the laser pulse and the generation of an intense broadband supercontinuum.

To illustrate this behavior, the nonlinear propagation of a 50 fs laser pulse with a pulse energy of $4 \mu \mathrm{J}$ conventionally focused into water is presented in Figure $4 \mathrm{a}$. In front of the focal plane $(\mathrm{z}=-200 \mu \mathrm{m})$, the intensity profile is similarly distributed in the linear $\left(\mathrm{E}_{\text {pulse }} \approx 0 \mu \mathrm{J}\right)$ and the nonlinear regime $\left(\mathrm{E}_{\text {pulse }}=4 \mu \mathrm{J}\right)$ resulting in a high intensity of $\sim 14 \mathrm{TW} / \mathrm{cm}^{2}$. During the propagation towards the focal plane $(\mathrm{z}=0 \mu \mathrm{m})$ self-steepening compresses the conventionally focused pulse to an even shorter duration of $30 \mathrm{fs}$. Downstream from the focal plane, the pulse is trapped within an elongated filament owing to the dynamical balance between Kerr self-focusing of the pulse and the defocusing refractive index diminution of the laser-induced plasma. Moreover, the defocusing effect of the plasma restricts the intensity to a maximum 
(a)

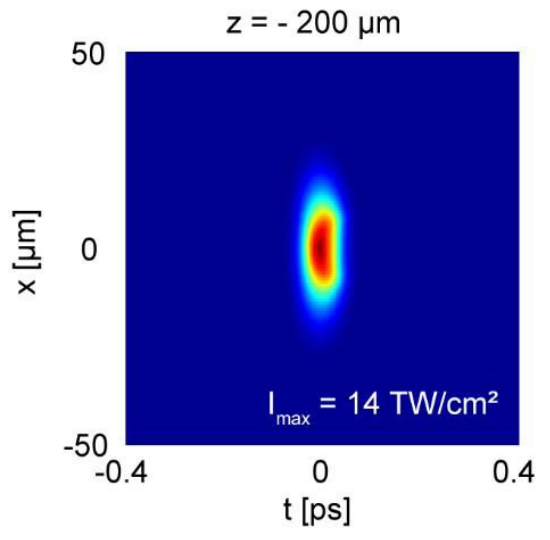

Conventional focusing

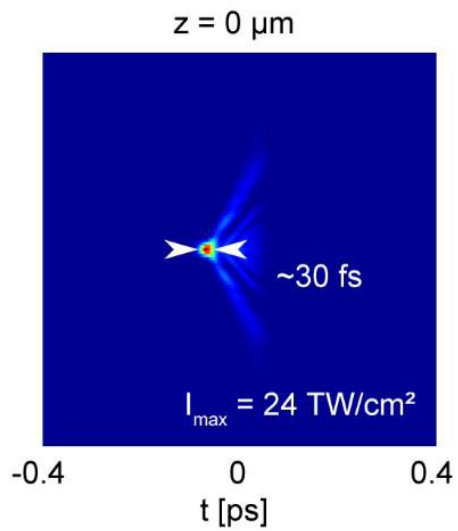

Simultaneous spatial and temporal focusing (b)

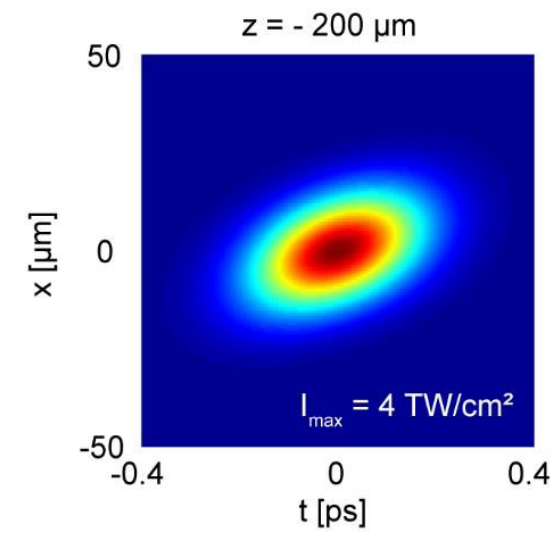

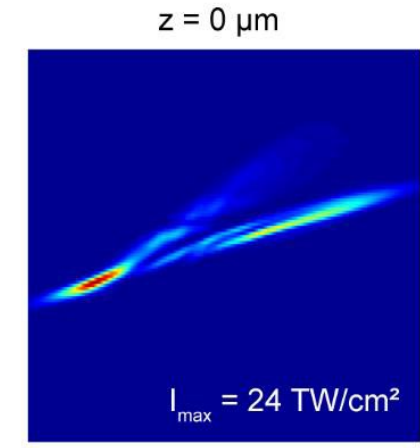

$-0.4$

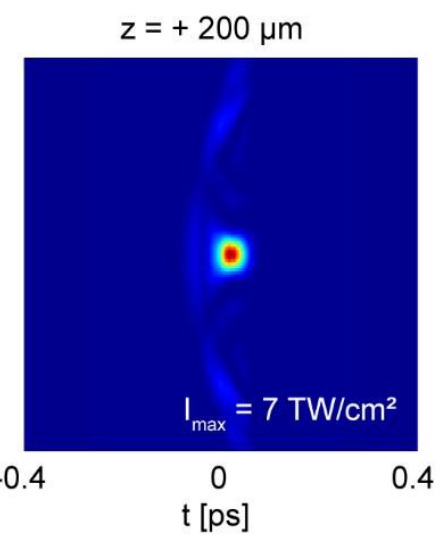

0.4

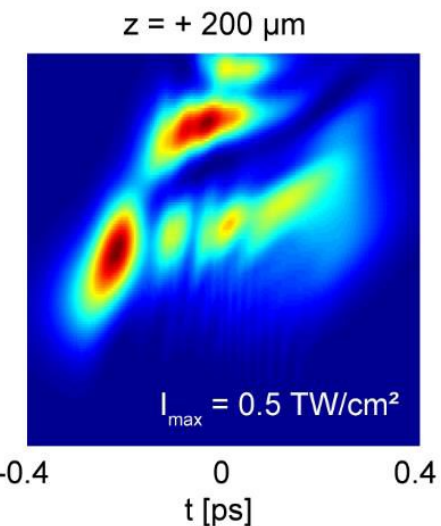

Figure 4. Simulation of the nonlinear pulse propagation $\left(E_{\text {pulse }} \approx 4 \mu \mathrm{J}\right)$ of pulses focused (a) conventionally and (b) using SSTF, showing $x / t$-intensity profiles nearby the focal plane $(z=-200 \mu \mathrm{m}, 0 \mu \mathrm{m},+200 \mu \mathrm{m})$.

value of $24 \mathrm{TW} / \mathrm{cm}^{2}$, referred to as 'intensity clamping'. ${ }^{7,}{ }^{29}$ Although $\sim 65 \%$ of the incident pulse energy is absorbed or scattered by the plasma, filamentation hampers the divergence of the pulse and keeps the intensity at a comparatively high level of $\sim 7 \mathrm{TW} / \mathrm{cm}^{2}$ even $200 \mu \mathrm{m}$ downstream from the focal plane.

In the nonlinear SSTF pulse propagation (Figure 4b), the intensity profile in front of the focal plane $(\mathrm{z}=-200 \mu \mathrm{m})$ is similar to the linear regime shown above (compare Figure $3 b)$. At the focal plane $(\mathrm{z}=0 \mu \mathrm{m})$ the intensity profile becomes distorted, whereas the fundamental characteristics of the SSTF pulse are conserved. Thus, the PFT remains similar to the linear case and the pulse is locally compressed to $\sim 50 \mathrm{fs}$. Owing to the intensity clamping effect mentioned above, a maximum intensity similarly achieved by conventional focusing is detected. Downstream from the focus, the increase of the pulse duration impedes the filamentation of the pulse and enables its divergence. Consequently, the intensity downstream from the focal plane is significantly reduced by both the spatial divergence and temporal broadening of the SSTF pulse.

Owing to the intensity reduction outside of the focal region, SSTF is able to reduce detrimental nonlinear side-effects and, furthermore, provides an additional degree of freedom via shaping the variation of the pulse duration during the pulse propagation. Consequently, numerous publications have shown its potential for improved laser materials processing. For example, Vitek et al. demonstrated the ability of backside-processing of $6 \mathrm{~mm}$ thick fused silica avoiding adverse selffocusing, filamentation and supercontinuum generation. ${ }^{30}$ Moreover, the ablation of bone with nearly parallel sidewalls under aqueous immersion was shown experimentally in this study, proposing this technique as a versatile tool for surgical purposes such as craniotomies. Furthermore, He et al. demonstrated theoretically and experimentally the formation of spherically shaped intensity distributions at the SSTF focus. ${ }^{31}$ Using this technique microfluidic channels with a circular cross-sectional shape were generated in fused silica. To overcome the effect of intensity clamping discussed above, SSTF was further applied to enhance the intensity inside the filament in air. ${ }^{32}$ While the length of the filament was reduced by a 
factor of 25 , this technique was proposed to provide high-order harmonic generation with improved conversion efficiency and higher cutoff photon energies. Furthermore, spatio-temporal focusing has been combined with patterned excitation for purposes of multiphoton microfabrication of polymer structures. ${ }^{33}$ Using a deformable mirror device, arbitrarily shaped 2D-patterns were imaged into the focal spot of the SSTF setup, providing a fabrication resolution of $\sim 1 \mu \mathrm{m}$ and enabling a stacking of $2 \mathrm{D}$-structures.

Next to manufacturing techniques, SSTF has also been studied for purposes of medical applications. Investigating tissue ablation by means of SSTF and conventional focusing, Block et al. showed a reduced focal plane upstream shift, reduced filamentation and weaker bubble formation using SSTF. ${ }^{34}$ Moreover, investigations realized by our research group showed the large potential of SSTF for intraocular fs-laser surgery, e.g. to provide minimally-invasive cuts within transparent tissue such as the crystalline lens or the vitreous body. ${ }^{23}$ In this study, the nonlinear pulse propagation in aqueous media was observed comparing SSTF and conventional focusing using equivalent focusing conditions. To outline the main findings, Figure 5 presents microscope images of fs-laser-induced modifications within gelatine, which serves as a model system for human lens tissue. For conventional focusing, ultrashort laser pulses with a pulse duration of $50 \mathrm{fs}$ centered at a wavelength of $800 \mathrm{~nm}$ are focused by means of a $25 \mathrm{~mm}$-off-axis parabolic mirror into the sample. Owing to the approximately circular beam diameter with $2 \mathrm{w}_{\mathrm{x}} \approx 2 \mathrm{w}_{\mathrm{y}} \approx 5 \mathrm{~mm}$, the NA was 0.1 as discussed in the numerical study above. Since for both conventional focusing and SSTF underfilling the aperture of the focusing lens can increase detrimental effects such as self-focusing and filamentation, ${ }^{35}$ the focusing conditions are optimized similarly for both configurations. Thus, for SSTF a cylinder lens telescope was used to reduce the horizontal beam width in front of the grating configuration by a factor of 5 to an elliptical beam size of $2 \mathrm{w}_{\mathrm{x}, \mathrm{in}} \approx 1 \mathrm{~mm}$ and $2 \mathrm{w}_{\mathrm{y}} \approx 5 \mathrm{~mm}$. Thereafter, the elliptical beam is spectrally separated by means of a grating setup similar to the one shown in Figure 2. The grating configuration provides a spatial chirp rate $\beta_{\mathrm{BA}} \approx 5$. Thus, the spectral separation results in stretching the input beam horizontally to a width of $2 \mathrm{w}_{\mathrm{x}, \mathrm{SSTF}} \approx 5$ $\mathrm{mm}$, yielding a circular beam size and NA similar to the conventional focusing configuration discussed above. Because $\beta_{\mathrm{BA}} \approx 5$, the pulse duration outside of the focal region is increased to $\tau_{\mathrm{SSTF}} \approx 250 \mathrm{fs}$. At the focal plane the pulse is compressed to the bandwidth-limited local pulse duration of $\sim 50 \mathrm{fs}$ (see inset of Figure 2). When the SSTF pulses are focused into the gelatine sample with a low pulse energy of $1 \mu \mathrm{J}$, the laser-induced disruptions are well confined to the focal plane for both focusing conditions (Figure 5a and 5b), although disruptions induced with SSTF show a reduction in length by a factor of $\sim 2$. For a high pulse energy of $8 \mu \mathrm{J}$, Kerr self-focusing and filamentation of the conventionally focused pulse cause the elongation of the disruption to a length of $>900 \mu \mathrm{m}$ (Figure 5c). Moreover, intense side-branches and luminance fluctuations within the central channel are observed, which may be attributed to multi-filamentation of the pulse. ${ }^{36}$ Hence, strong fluctuations of the plasma density within the filaments cause the breakup of the central disruption into a multitude of separated disruptions as well as the chemical decomposition of the molecular bonds of the tissue. ${ }^{37}$ In agreement with the numerical analysis presented above, the modifications induced by SSTF pulses with $8 \mu \mathrm{J}$ are more homogeneous and are not showing side-branches or breakups (Figure 5d). Owing to the localization of the ultrashort pulse duration, detrimental nonlinear side-effects such as self-focusing, filamentation and self-phase modulation are avoided, which restricts the nonlinear interactions and the optical breakdown to the focal region.

(a) Conventional focusing, $1 \mu \mathrm{J}$

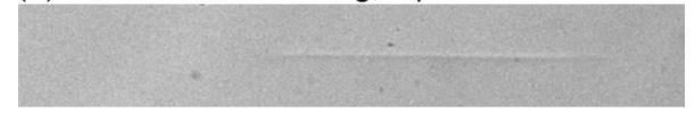

(c) Conventional focusing, $8 \mu \mathrm{J}$ (b) SSTF, $1 \mu \mathrm{J}$
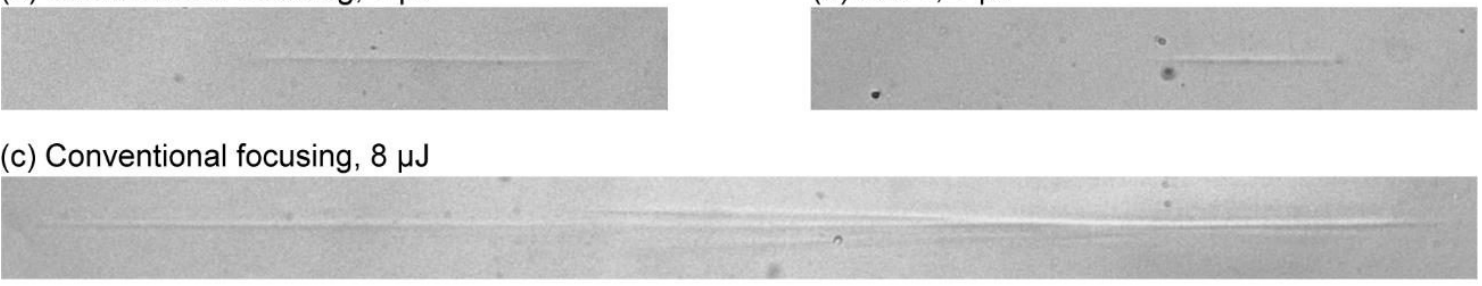

(d) SSTF, $8 \mu \mathrm{J}$ 


\section{CONCLUSION}

Owing to the opportunity of accurately tailoring the laser-material interaction, ultrashort fs-laser pulses have enabled materials processing with high precision and low side-effects. Moreover, based on the development of compact and reliable fs-laser sources, innovative applications such as multiphoton fluorescence microscopy, processing of transparent materials and medical surgery gain even more importance. However, for several applications the nonlinear material interactions caused by conventional focusing are limited, since the intensity distribution at the focal plane cannot be freely designed, e.g. owing to the physical correlation between numerical aperture, focal spot size and the Rayleigh length. Here, simultaneous spatial and temporal focusing provides an additional degree of freedom, since the evolution of the pulse duration during the pulse propagation towards the focal plane can be adjusted to the targeted nonlinear pulse-material interactions. Hence, SSTF has been widely applied in multiphoton microscopy due to its ability to combine wide-field illumination with a high spatial resolution up to few $\mu \mathrm{m}$. Additionally, the localization of the region of maximum intensity towards the focal plane enables reducing detrimental nonlinear interactions such as Kerr self-focusing, self-phase modulation and filamentation in order to improve the confinement of laser-induced modifications. Finally, innovative procedures such as 2D-SSTF or incorporating SSTF into conventional chirped pulse amplifier systems aim at even stronger intensity confinement and simplified application of this technique to provide high-precision processing of transparent materials such as intraocular fs-surgery.

\section{ACKNOWLEDGEMENTS}

This study was supported by the Thuringian Ministry of Education, Science and Culture (OptiMi 2020-Graduate Research School 'Green Photonics', B514-10061), the German Research Foundation (Leibniz program) and the Carl Zeiss Foundation. The numerical simulations were performed at the Rechenzentrum Garching.

\section{REFERENCES}

[1] Cheng, J., Liu, C.S., Shang, S., Liu, D., Perrie, W., Dearden, G. and Watkins, K., "A review of ultrafast laser materials micromachining," Opt. Laser. Technol. 46, 88-102 (2013).

[2] Chichkov, B.N., Momma, C., Nolte, S., von Alvensleben, F. and Tünnermann, A., "Femtosecond, picosecond and nanosecond laser ablation of solids," Appl. Phys. A 63(2), 109-115 (1996).

[3] Itoh, K., Watanabe, W., Nolte, S. and Schaffer, C.B., "Ultrafast processes for bulk modification of transparent materials," MRS Bull. 31(8), 620-625 (2006).

[4] Sugioka, K. and Cheng, Y., "Femtosecond laser three-dimensional micro- and nanofabrication," Appl. Phys. Rev. 1(4), 041303 (2014).

[5] Merker, M., Ackermann, R., Kammel, R., Kunert, K.S. and Nolte, S., "An in vitro study on focusing fs-laser pulses into ocular media for ophthalmic surgery," Lasers Surg. Med. 45(9), 589-596 (2013).

[6] Ackermann, R., Kunert, K.S., Kammel, R., Bischoff, S., Bühren, S.C., Schubert, H., Blum, M. and Nolte, S., "Femtosecond laser treatment of the crystalline lens: a 1-year study of possible cataractogenesis in minipigs," Graefes Arch. Clin. Exp. Ophthalmol. 249(10), 1567-1573 (2011).

[7] Couairon, A. and Mysyrowicz, A., "Femtosecond filamentation in transparent media," Phys. Rep. 441(2-4), 47189 (2007).

[8] Kammel, R., Ackermann, R., Tünnermann, A. and Nolte, S., "Pump-probe investigation of fs-LIOB in water by simultaneous spatial and temporal focusing," Proc. SPIE 8611, 86110A (2013).

[9] Liu, W., Petit, S., Becker, A., Aközbek, N., Bowden, C.M. and Chin, S.L., "Intensity clamping of a femtosecond laser pulse in condensed matter," Opt. Commun. 202(1-3), 189-197 (2002).

[10] Zhu, G., van Howe, J., Durst, M., Zipfel, W. and Xu, C., "Simultaneous spatial and temporal focusing of femtosecond pulses," Opt. Express 13(6), 2153-2159 (2005).

[11] Oron, D., Tal, E. and Silberberg, Y., "Scanningless depth-resolved microscopy," Opt. Express 13(5), 1468-1476 (2005).

[12] Durfee, C.G., Greco, M., Block, E., Vitek, D. and Squier, J.A., "Intuitive analysis of space-time focusing with double-ABCD calculation," Opt. Express 20(13), 14244-14259 (2012). 
[13] Papagiakoumou, E., de Sars, V., Oron, D. and Emiliani, V., "Patterned two-photon illumination by spatiotemporal shaping of ultrashort pulses," Opt. Express 16(26), 22039-22047 (2008).

[14] Therrien, O.D., Aube, B., Pages, S., Koninck, P.D. and Cote, D., "Wide-field multiphoton imaging of cellular dynamics in thick tissue by temporal focusing and patterned illumination," Biomed. Opt. Express 2(3), 696-704 (2011).

[15] Papagiakoumou, E., de Sars, V., Emiliani, V. and Oron, D., "Temporal focusing with spatially modulated excitation," Opt. Express 17(7), 5391-5401 (2009).

[16] Leshem, B., Hernandez, O., Papagiakoumou, E., Emiliani, V. and Oron, D., "When can temporally focused excitation be axially shifted by dispersion?," Opt. Express 22(6), 7087-7098 (2014).

[17] Straub, A., Durst, M.E. and Xu, C., "High speed multiphoton axial scanning through an optical fiber in a remotely scanned temporal focusing setup," Biomed. Opt. Express. 2(1), 80-88 (2011).

[18] Song, Q., Nakamura, A., Hirosawa, K., Isobe, K., Midorikawa, K. and Kannari, F., "Two-dimensional spatiotemporal focusing of femtosecond pulses and its applications in microscopy," Rev. Sci. Instrum. 86(8), 083701 (2015).

[19] Squier, J., Thomas, J., Block, E., Durfee, C. and Backus, S., "High average power Yb:CaF femtosecond amplifier with integrated simultaneous spatial and temporal focusing for laser material processing," Appl. Phys. A 114(1), 209-214 (2014).

[20] Block, E., Thomas, J., Durfee, C. and Squier, J., "Integrated single grating compressor for variable pulse front tilt in simultaneously spatially and temporally focused systems," Opt. Lett. 39(24), 6915-6918 (2014).

[21] Durst, M.E., Zhu, G. and Xu, C., "Simultaneous Spatial and Temporal Focusing in Nonlinear Microscopy," Opt. Commun. 281(7), 1796-1805 (2008).

[22] Zhang, S., Asoubar, D., Kammel, R., Nolte, S. and Wyrowski, F., "Analysis of pulse front tilt in simultaneous spatial and temporal focusing," J. Opt. Soc. Am. A 31(11), 2437-2446 (2014).

[23] Kammel, R., Ackermann, R., Thomas, J., Götte, J., Skupin, S., Tünnermann, A. and Nolte, S., "Enhancing precision in fs-laser material processing by simultaneous spatial and temporal focusing," Light-Sci. Appl. 3(5), e169 (2014).

[24] Coughlan, M.A., Plewicki, M. and Levis, R.J., "Parametric spatio-temporal control of focusing laser pulses," Opt. Express 17(18), 15808-15820 (2009).

[25] He, F., Zeng, B., Chu, W., Ni, J., Sugioka, K., Cheng, Y. and Durfee, C.G., "Characterization and control of peak intensity distribution at the focus of a spatiotemporally focused femtosecond laser beam," Opt. Express 22(8), 9734 (2014).

[26] Wang, Z., He, F., Ni, J., Jing, C., Xie, H., Zeng, B., Chu, W., Qiao, L. and Cheng, Y., "Interferometric characterization of pulse front tilt of spatiotemporally focused femtosecond laser pulses," Opt. Express 22(21), 26328 (2014).

[27] Kazansky, P.G., Yang, W.J., Bricchi, E., Bovatsek, J., Arai, A., Shimotsuma, Y., Miura, K. and Hirao, K., ""Quill" writing with ultrashort light pulses in transparent materials," Appl. Phys. Lett. 90(15), 151120 (2007).

[28] Vitek, D.N., Block, E., Bellouard, Y., Adams, D.E., Backus, S., Kleinfeld, D., Durfee, C.G. and Squier, J.A., "Spatio-temporally focused femtosecond laser pulses for nonreciprocal writing in optically transparent materials," Opt. Express 18(24), 24673-24678 (2010).

[29] Liu, W., Petit, S., Becker, A., Akozbek, N., Bowden, C.M. and Chin, S.L., "Intensity clamping of a femtosecond laser pulse in condensed matter," Opt. Commun. 202(1-3), 189-197 (2002).

[30] Vitek, D.N., Adams, D.E., Johnson, A., Tsai, P.S., Backus, S., Durfee, C.G., Kleinfeld, D. and Squier, J.A., "Temporally focused femtosecond laser pulses for low numerical aperture micromachining through optically transparent materials," Opt. Express 18(17), 18086-18094 (2010).

[31] He, F., Xu, H., Cheng, Y., Ni, J.L., Xiong, H., Xu, Z.Z., Sugioka, K. and Midorikawa, K., "Fabrication of microfluidic channels with a circular cross section using spatiotemporally focused femtosecond laser pulses," Opt. Lett. 35(7), 1106-1108 (2010).

[32] Zeng, B., Chu, W., Gao, H., Liu, W.W., Li, G.H., Zhang, H.S., Yao, J.P., Ni, J.L., Chin, S.L., Cheng, Y. and Xu, Z.Z., "Enhancement of peak intensity in a filament core with spatiotemporally focused femtosecond laser pulses," Phys. Rev. A 84(6), 063819 (2011).

[33] Li, Y.C., Cheng, L.C., Chang, C.Y., Lien, C.H., Campagnola, P.J. and Chen, S.J., "Fast multiphoton microfabrication of freeform polymer microstructures by spatiotemporal focusing and patterned excitation," Opt. Express 20(17), 19030-19038 (2012). 
[34] Block, E., Greco, M., Vitek, D., Masihzadeh, O., Ammar, D.A., Kahook, M.Y., Mandava, N., Durfee, C. and Squier, J., "Simultaneous spatial and temporal focusing for tissue ablation," Biomed. Opt. Express 4(6), 831-841 (2013).

[35] Cheng, Y., Xie, H.Q., Wang, Z.H., Li, G.H., Zeng, B., He, F., Chu, W., Yao, J.P. and Qiao, L.L., "Onset of nonlinear self-focusing of femtosecond laser pulses in air: Conventional vs spatiotemporal focusing," Phys. Rev. A 92(2), 023854 (2015).

[36] Skupin, S. and Bergé, L., "Modeling ultrashort filaments of light," Discret. Contin. Dyn. S 23(4), 1099-1139 (2008).

[37] Vogel, A., Noack, J., Hüttman, G. and Paltauf, G., "Mechanisms of femtosecond laser nanosurgery of cells and tissues," Appl. Phys. B 81(8), 1015-1047 (2005). 